\title{
NON-MARINE AQUATIC MOLLUSCS (GASTROPODA, BIVALVIA) OF RAB ISLAND (CROATIA)
}

\author{
LUBOŠ BERAN \\ Nature Conservation Agency of the Czech Republic, Regional Office Kokořínsko - Máchův kraj \\ Protected Landscape Area Administration, Česká 149, CZ-276 01 Mělník, Czech Republic \\ (e-mail: lubos.beran@nature.cz)
}

Beran, L.: Non-marine aquatic molluscs (Gastropoda, Bivalvia) of Rab Island (Croatia). Nat. Croat., Vol. 24, No. 2., 255-264, 2015, Zagreb.

This paper presents the results of probably the first rather detailed survey of aquatic molluscs of Rab Island. This island belongs among those Croatian islands situated in the northern Adriatic Sea. Altogether 11 species of aquatic non-marine molluscs (9 gastropods, 2 bivalves) were found at 49 sites in 2012-2015. Two species recorded, Ecrobia ventrosa and Myosotella myosotis, inhabit brackish waters while the other species belong among freshwater molluscs and were found in springs, wells, ditches, rivulets and small brooks. Kerkia kareli, a species described in 2014 from Pag Island which inhabits phreatic waters, was found at one site. The non-native Physella acuta was recorded from many sites and this find is probably the first known occurrence of a non-native aquatic mollusc at least from the northern Croatian islands. The results of this research were compared with aquatic molluscan assemblages of other Croatian islands.

Key words: Mollusca, Gastropoda, Bivalvia, Kerkia kareli, Rab Island, Croatia, faunistics

Beran, L.: Nemorski vodeni mekušci (Gastropoda, Bivalvia) otoka Raba (Hrvatska). Nat. Croat., Vol. 24, No. 2., 255-264, 2015, Zagreb.

Rad donosi rezultate vjerojatno prvog detaljnijeg istraživanja slatkovodnih mekušaca otoka Raba. Taj otok pripada jadranskim otocima smještenim u sjevernom Jadranu. Tijekom 2012-2015 na 49 lokaliteta pronađeno je sveukupno 11 vrsta slatkovodnih mekušaca ( 9 puževa, 2 školjkaša). Dvije vrste, Ecrobia ventrosa i Myosotella myosotis nastanjuju bočate vode, dok ostale vrste pripadaju slatkovodnim mekušcima i nađeni su u izvorima, bunarima, jarcima, malim vodenim tokovima i potočićima. Kerkia kareli, vrsta zabilježena 2014. na otoku Pagu, koja nastanjuje freatičke vode, nađena je na jednom lokalitetu. Alohtona vrsta Physella acuta je zabilježena na mnogo lokaliteta, i to je vjerojatno prvo poznato pojavljivanje alohtonog vodenog mekušca barem što se tiče sjevernojadranskih otoka. Rezultati istraživanja se uspoređuju sa zajednicama vodenih mekušaca drugih jadranskih otoka.

Ključne riječi: Mollusca, Gastropoda, Bivalvia, Kerkia kareli, otok Rab, Hrvatska, faunistika

\section{INTRODUCTION}

$\mathrm{Rab}$ is an island of the Croatian coast situated in the northern Adriatic Sea. Aquatic molluscs of this island are poorly known and previously to this research no inventory of non-marine aquatic molluscs had been made. Only the occurrence of Pseudamnicola conovula at this island is mentioned in RADOMAN (1983). Therefore, the main aim of this research was to provide an inventory of aquatic molluscs inhabiting this island.

\section{MATERIAL AND METHODS}

Data were obtained from field surveys conducted in the years 2012-2015. Only nonmarine aquatic (freshwater, brackish) molluscs were studied. Altogether 49 sites with the 
occurrence of aquatic molluscs were studied and these sites contain springs, wells, ditches, rivulets and small brooks and also brackish canals and lagoons. The main sampling method used for field research was the washing of vegetation or sediments using a metal sieve (a kitchen strainer, diameter $20 \mathrm{~cm}$, mesh size $0.8 \mathrm{~mm}$ ) combined with collection by hand after searching the surfaces of plants, stones, wood and artificial materials (e.g. plastic bags and bottles). Wells were studied using this sieve on 2.5-3 $\mathrm{m}$ long pole.

Molluscs were classified using various shell characteristics or dissected and then identified using their copulatory organs if identification based solely on the shell was impossible. Specimens for dissection were killed in hot water and then fixed in $70 \%$ ethanol. Selected materials of shells and killed specimens in $70 \%$ ethanol are deposited in the author's collection.

\section{STUDY AREA}

Rab is a Croatian island in the northern Adriatic Sea. The island is $22 \mathrm{~km}$ long (shoreline $103 \mathrm{~km}$ ) and has an area of $91 \mathrm{~km}^{2}$. The highest peak is Kamenjak at $410 \mathrm{~m}$. The northeastern side of the island is mostly barren, while the southwestern side is covered by one of the last oak forests of the Mediterranean. The lowest parts of the island are much more green and are crossed by many small and often also permanent watercourses.

\section{LIST OF INVESTIGATED SITES}

Data in the list are as follows: site number, geographical co-ordinates (http://itouchmap.com), name of the nearest settlement, description of the site, date of investigation. Sites are depicted at Fig. 1.

$1-44^{\circ} 50^{\prime} 25.4^{\prime \prime} \mathrm{N}, 14^{\circ} 43^{\prime} 28.2^{\prime \prime} \mathrm{E}$, Lopar, a small rivulet at the north edge of Lopar, 2.1.2015;

$2-44^{\circ} 50^{\prime} 20.2^{\prime \prime} \mathrm{N}, 14^{\circ} 43^{\prime} 27.3^{\prime \prime} \mathrm{E}$, Lopar, a small rivulet in Lopar ca $300 \mathrm{~m}$ upstream of its inflow into the sea, 2.1.2015;

$3-44^{\circ} 50^{\prime} 15.9^{\prime \prime} \mathrm{N}, 14^{\circ} 43^{\prime} 27.2^{\prime \prime} \mathrm{E}$, Lopar, a canal near the ferry in Lopar, 28.12.2012;

$4-44^{\circ} 49^{\prime} 47.3^{\prime \prime} \mathrm{N}, 14^{\circ} 43^{\prime} 46.9^{\prime \prime} \mathrm{E}$, Lopar, a brook by the supermarket (Konzum) in Lopar, 2.1.2015;

$5-44^{\circ} 49^{\prime} 54.7^{\prime \prime} \mathrm{N}, 14^{\circ} 44^{\prime} 07.5^{\prime \prime} \mathrm{E}$, Lopar, a small brook by the well Jerkovica in Lopar, 27.12.2013;

$6-44^{\circ} 49^{\prime} 51.1^{\prime \prime} \mathrm{N}, 14^{\circ} 44^{\prime} 03.6^{\prime \prime} \mathrm{E}$, Lopar, a brook overgrown by Cardamine sp. ca $100 \mathrm{~m}$ upstream of the road in Lopar, 27.12.2013;

$7-44^{\circ} 49^{\prime} 44.4^{\prime \prime} \mathrm{N}, 14^{\circ} 43^{\prime} 53.6^{\prime \prime} \mathrm{E}$, Lopar, a brook overgrown by Cardamine sp. ca $50 \mathrm{~m}$ downstream of the road in Lopar, 27.12.2013;

$8-44^{\circ} 49^{\prime} 39.4^{\prime \prime} \mathrm{N}, 14^{\circ} 43^{\prime} 44.1^{\prime \prime} \mathrm{E}$, Lopar, a brook upstream of the inflow of the small ditch in Lopar, 2.1.2015;

$9-44^{\circ} 49^{\prime} 40.1^{\prime \prime} \mathrm{N}, 14^{\circ} 43^{\prime} 40^{\prime \prime} \mathrm{E}$, Lopar, a brook by the road Lopar - Rab, 2.1.2015;

$10-44^{\circ} 49^{\prime} 35.8^{\prime \prime} \mathrm{N}, 14^{\circ} 43^{\prime} 40.5^{\prime \prime} \mathrm{E}$, Lopar, a small ditch ca $50 \mathrm{~m}$ upstream of its inflow into the brook in Lopar, 2.1.2015;

$11-44^{\circ} 49^{\prime} 30.9^{\prime \prime} \mathrm{N}, 14^{\circ} 45^{\prime} 02^{\prime \prime} \mathrm{E}$, Lopar, small pools in a dried brook ca $50 \mathrm{~m}$ upstream of the inflow to the sea, 27.12.2013;

$12-44^{\circ} 49^{\prime} 27.3^{\prime \prime} \mathrm{N}, 14^{\circ} 44^{\prime} 17.8^{\prime \prime} \mathrm{E}$, Lopar, a small ditch in reeds by the road to the camp in Lopar, 27.12.2013; 


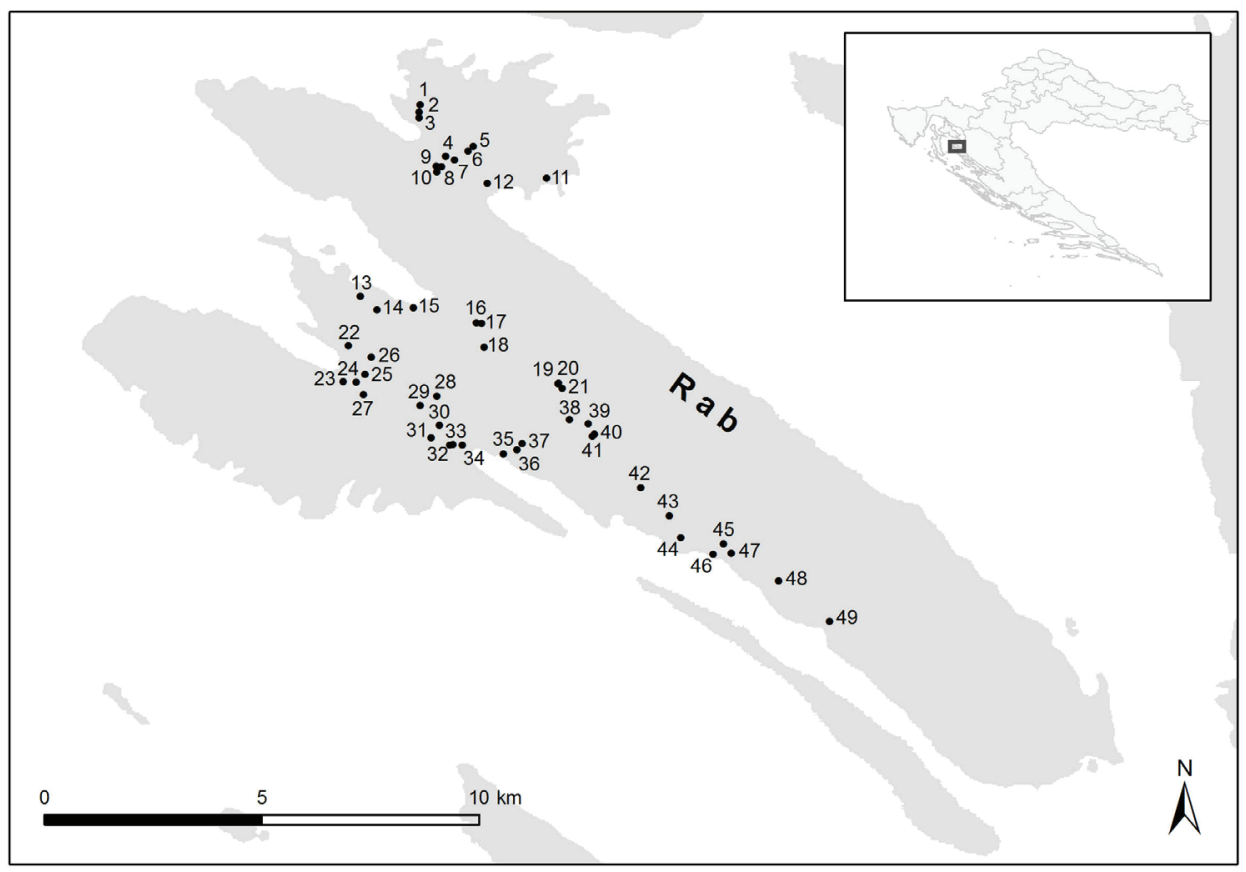

Fig. 1. The map of Rab Island with sites under study. Orig. H. Kaňková.

$13-44^{\circ} 48^{\prime} 03.3^{\prime \prime} \mathrm{N}, 14^{\circ} 42^{\prime} 43.7^{\prime \prime} \mathrm{E}$, Supetarska Draga, a small rivulet ca $50 \mathrm{~m}$ upstream of its inflow into the sea, 29.12.2014;

$14-44^{\circ} 47^{\prime} 53^{\prime \prime} \mathrm{N}, 14^{\circ} 42^{\prime} 56.1^{\prime \prime} \mathrm{E}$, Supetarska Draga, a spring and rivulet in the southwestern part of Donja Supetarska Draga, 29.12.2014;

$15-44^{\circ} 47^{\prime} 54.7^{\prime \prime} \mathrm{N}, 14^{\circ} 43^{\prime} 23^{\prime \prime} \mathrm{E}$, Supetarska Draga, a brackish pool by the sea in Donja Supetarska Draga, 30.12.2012;

$16-44^{\circ} 47^{\prime} 43.5^{\prime \prime} \mathrm{N}, 14^{\circ} 44^{\prime} 10^{\prime \prime} \mathrm{E}$, Supetarska Draga, a small ditch by the local road ca $500 \mathrm{~m}$ northwest of the church Sv. Petar, 27.12.2013;

$17-44^{\circ} 47^{\prime} 43^{\prime \prime} \mathrm{N}, 14^{\circ} 44^{\prime} 13.8^{\prime \prime} \mathrm{E}$, Supetarska Draga, a small ditch ca $450 \mathrm{~m}$ northwest of the church Sv. Petar, 27.12.2013;

$18-44^{\circ} 47^{\prime} 25.4^{\prime \prime} \mathrm{N}, 14^{\circ} 44^{\prime} 15.6^{\prime \prime} \mathrm{E}$, Supetarska Draga, a small brook near Supetarska Draga by the bridge of the local road near the church Sv. Petar, 28.12.2012;

$19-44^{\circ} 46^{\prime} 58.3^{\prime \prime} \mathrm{N}, 14^{\circ} 45^{\prime} 10.8^{\prime \prime} \mathrm{E}$, Supetarska Draga, a well by the local road ca $1100 \mathrm{~m}$ southeast of the church Sv. Petar, 27.12.2013;

$20-44^{\circ} 46^{\prime} 58.4^{\prime \prime} \mathrm{N}, 14^{\circ} 45^{\prime} 10.9^{\prime \prime} \mathrm{E}$, Supetarska Draga, a small rivulet by the local road and by the well ca $1100 \mathrm{~m}$ southeast of the church Sv. Petar, 27.12.2013;

$21-44^{\circ} 46^{\prime} 54.6^{\prime \prime} \mathrm{N}, 14^{\circ} 45^{\prime} 13.9^{\prime \prime} \mathrm{E}$, Supetarska Draga, a small ditch by the local road ca 1200 m southeast of the church Sv. Petar, 27.12.2013;

$22-44^{\circ} 47^{\prime} 26.3^{\prime \prime} \mathrm{N}, 14^{\circ} 42^{\prime} 34.5^{\prime \prime} \mathrm{E}$, Kampor, a small rivulet ca $50 \mathrm{~m}$ upstream of its inflow into the sea in Kampor, 28.12.2014;

$23-44^{\circ} 46^{\prime} 59.7^{\prime \prime} \mathrm{N}, 14^{\circ} 42^{\prime} 30.7^{\prime \prime} \mathrm{E}$, Kampor, a brook ca $100 \mathrm{~m}$ upstream of the inflow to the sea, a) 27.12.2012, b) 30.12.2013; 
$24-44^{\circ} 46^{\prime} 59.2^{\prime \prime} \mathrm{N}, 14^{\circ} 42^{\prime} 40.4^{\prime \prime} \mathrm{E}$, Kampor, a small brook by the cemetery in Kampor, 30.12.2013;

$25-44^{\circ} 47^{\prime} 05^{\prime \prime} \mathrm{N}, 14^{\circ} 42^{\prime} 47.2^{\prime \prime} \mathrm{E}$, Kampor, a small rivulet by the road in the northwest part of Kampor, a) 27.12.2012, b) 1.1.2015;

$26-44^{\circ} 47^{\prime} 17.8^{\prime \prime} \mathrm{N}, 14^{\circ} 42^{\prime} 51.8^{\prime \prime} \mathrm{E}$, Kampor, a small brook in the forest on the north edge of Kampor, 31.12.2014;

$27-44^{\circ} 46^{\prime} 50^{\prime \prime} \mathrm{N}, 14^{\circ} 42^{\prime} 45.8^{\prime \prime} \mathrm{E}$, Kampor, a small pool in pastures near the cemetery on the northwestern edge of Kampor, 31.12.2012;

$28-44^{\circ} 46^{\prime} 48.8^{\prime \prime} \mathrm{N}, 14^{\circ} 43^{\prime} 40.3^{\prime \prime} \mathrm{E}$, Kampor, a small spring and pools in valley north of the road Kampor-Rab, 28.12.2013;

$29-44^{\circ} 46^{\prime} 42^{\prime \prime} \mathrm{N}, 14^{\circ} 43^{\prime} 28^{\prime \prime} \mathrm{E}$, Kampor, a small brook by the bridge of the road Rab Kampor near the psychiatric clinic, 29.12.2012;

$30-44^{\circ} 46^{\prime} 27.3^{\prime \prime} \mathrm{N}, 14^{\circ} 43^{\prime} 42.3^{\prime \prime} \mathrm{E}$, Kampor, a small brook along the local road in Kampor, 28.12.2013;

$31-44^{\circ} 46^{\prime} 17.9^{\prime \prime} \mathrm{N}, 14^{\circ} 43^{\prime} 36^{\prime \prime} \mathrm{E}$, Kampor, a canal in fields on the southeast edge of Kampor, 28.12.2012;

$32-44^{\circ} 46^{\prime} 12.6^{\prime \prime} \mathrm{N}, 14^{\circ} 43^{\prime} 50.1^{\prime \prime} \mathrm{E}$, Kampor, a brook overgrown by reeds ca $30 \mathrm{~m}$ upstream of the road Kampor - Suha Punta, 28.12.2013;

$33-44^{\circ} 46^{\prime} 12.8^{\prime \prime} \mathrm{N}, 14^{\circ} 43^{\prime} 52.4^{\prime \prime} \mathrm{E}$, Kampor, a small canal by the bridge of the road Kampor - Suha Punta, 28.12.2013;

$34-44^{\circ} 46^{\prime} 12.5^{\prime \prime} \mathrm{N}, 14^{\circ} 43^{\prime} 59.4^{\prime \prime} \mathrm{E}$, Kampor, a small ditch by the sea in Kampor, 1.1.2015;

$35-44^{\circ} 46^{\prime} 05.9^{\prime \prime} \mathrm{N}, 14^{\circ} 44^{\prime} 29.9^{\prime \prime} \mathrm{E}$, Kampor, a small lagoon ca $50 \mathrm{~m}$ from the inflow of the brook into the sea by the St Euphemia Monastery, 1.1.2015;

$36-44^{\circ} 46^{\prime} 09^{\prime \prime} \mathrm{N}, 14^{\circ} 44^{\prime} 40.2^{\prime \prime} \mathrm{E}$, Kampor, a brook ca $250 \mathrm{~m}$ upstream of its inflow into the sea, 1.1.2015;

$37-44^{\circ} 46^{\prime} 13.7^{\prime \prime} \mathrm{N}, 14^{\circ} 44^{\prime} 44^{\prime \prime} \mathrm{E}$, Palit, a small ditch by the road between Kampor and Palit, a) 29.12.2012, b) 30.12.2013;

$38-44^{\circ} 46^{\prime} 31.6^{\prime \prime} \mathrm{N}, 14^{\circ} 45^{\prime} 19.3^{\prime \prime} \mathrm{E}$, Mundanije, a small brook by the bridge of the road Mundanije - Supetarska Draga, 28.12.2012;

$39-44^{\circ} 46^{\prime} 28.3^{\prime \prime} \mathrm{N}, 14^{\circ} 45^{`} 33.2^{\prime \prime} \mathrm{E}$, Mundanije, a small rivulet in Mundanije, 1.1.2015;

$40-44^{\circ} 46^{\prime} 20.5^{\prime \prime} \mathrm{N}, 14^{\circ} 45^{\prime} 37.7^{\prime \prime} \mathrm{E}$, Mundanije, a small rivulet upstream of its inflow into the brook in Mundanije, 29.12.2014;

$41-44^{\circ} 46^{\prime} 19^{\prime \prime} \mathrm{N}, 14^{\circ} 45^{\prime} 36^{\prime \prime} \mathrm{E}$, Mundanije, a small brook on the northwestern edge of Mundanije, 30.12.2012;

$42-44^{\circ} 45^{\prime} 40.8^{\prime \prime} \mathrm{N}, 14^{\circ} 46^{\prime} 12.3^{\prime \prime} \mathrm{E}$, Banjol, a brook by the road Rab - Lopar in Banjol, 29.12.2014;

$43-44^{\circ} 45^{\prime} 19.9^{\prime \prime} \mathrm{N}, 14^{\circ} 46^{\prime} 33.3^{\prime \prime} \mathrm{E}, \mathrm{Rab}$, a brook by the bridge of the road Rab - Barbat in Rab, a) 30.12.2012, b) 29.12.2013;

$44-44^{\circ} 45^{\prime} 03.5^{\prime \prime} \mathrm{N}, 14^{\circ} 46^{\prime} 42^{\prime \prime} \mathrm{E}$, Banjol, a brook flowing from Mundanije ca $50 \mathrm{~m}$ upstream of the inflow to the sea, 29.12.2013;

$45-44^{\circ} 44^{\prime} 58.9^{\prime \prime} \mathrm{N}, 14^{\circ} 47^{\prime} 13.7^{\prime \prime} \mathrm{E}$, Banjol, a small rivulet by the road Barbat - Rab in Banjol, 26.12.2013;

$46-44^{\circ} 44^{\prime} 51^{\prime \prime} \mathrm{N}, 14^{\circ} 47^{\prime} 06^{\prime \prime} \mathrm{E}$, Banjol, a small brook ca $30 \mathrm{~m}$ upstream of the inflow to the sea, 29.12.2013;

$47-44^{\circ} 44^{\prime} 52^{\prime \prime} \mathrm{N}, 14^{\circ} 47^{\prime} 19.4^{\prime \prime} \mathrm{E}$, Banjol, a small rivulet by the petrol station by the road Rab - Barbat, 29.12.2013; 

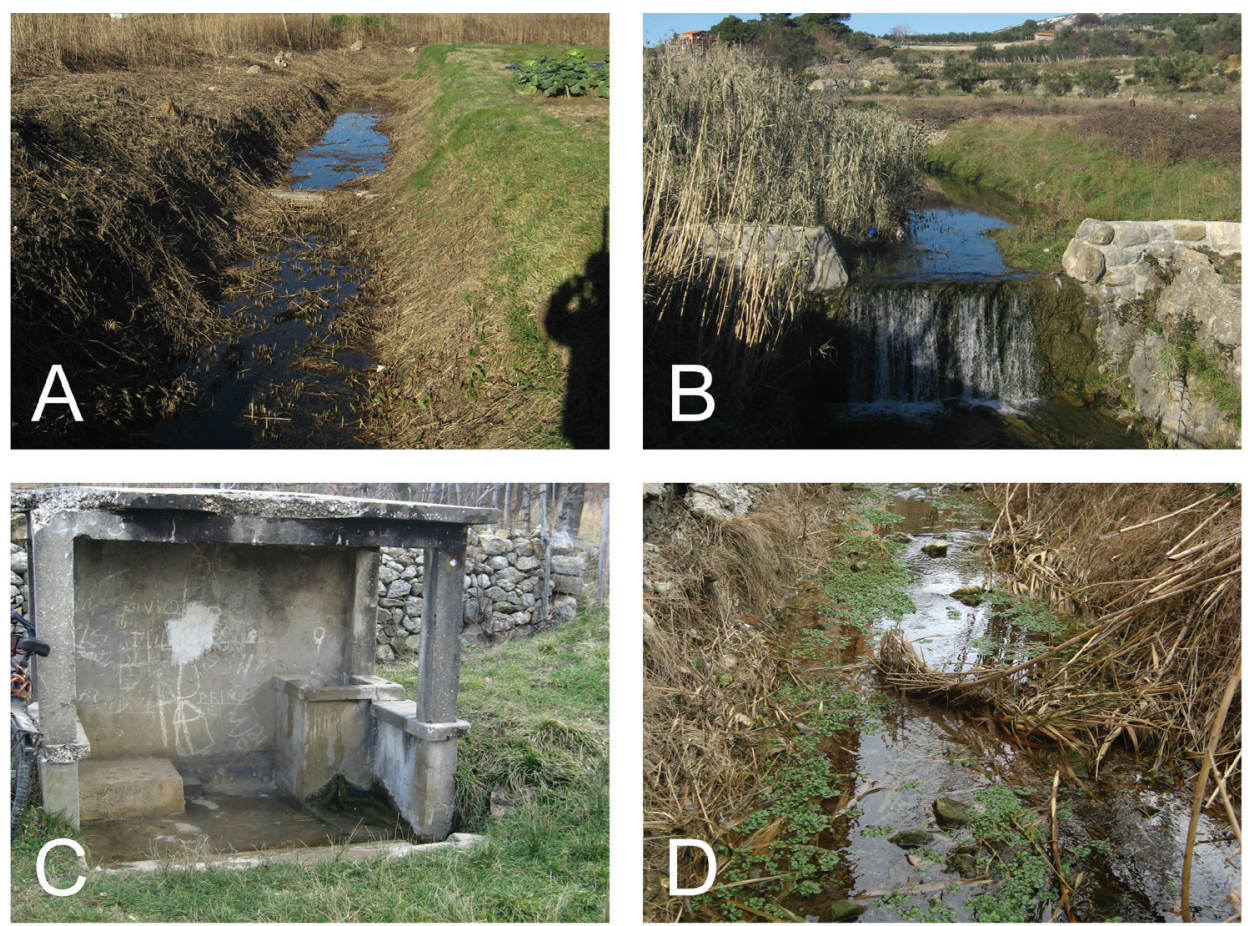

Fig. 2. Selected studied sites. A: a small ditch by the road between Kampor and Palit (site 37); B: a brook by the bridge of the road Rab - Barbat in Rab (site 43); C: a well by the local road ca $1100 \mathrm{~m}$ southeast of the church Sv. Petar (site 19); D: a small brook ca $30 \mathrm{~m}$ upstream of the inflow to the sea (site 46). Photo L. Beran.

$48-44^{\circ} 44^{\prime} 31.4^{\prime \prime} \mathrm{N}, 14^{\circ} 47^{\prime} 54.9^{\prime \prime} \mathrm{E}$, Barbat, a small pool in the small and dried rivulet by the road Barbat - Rab in Barbat, 26.12.2013;

$49-44^{\circ} 44^{\prime} 01.3^{\prime \prime} \mathrm{N}, 14^{\circ} 48^{\prime} 32.6^{\prime \prime} \mathrm{E}$, Barbat, a small rivulet by the road in Barbat, 30.12.2014;

\section{RESULTS}

The first rather detailed inventory of aquatic non-marine molluscs of the Rab Island confirmed the occurrence of 11 species (9 gastropods, 2 bivalves) at 49 sites. A list of molluscs found at particular sites and their density is given in Tab. 1-2.

Some sites (brackish habitats, several upper sections of small rivulets, ditches) with the positive occurrence of aquatic molluscs were inhabited by only one or two species (Tab. 1-2) while some brooks and rivulets were inhabited by more diversified molluscan assemblages with 3-6 species, such as Pseudamnicola conovula, Galba truncatula, Radix labiata, Physella acuta and less often also Pisidium personatum or P. casertanum. Galba truncatula was often found in ditches together with Anisus spirorbis. Kerkia kareli is a recently described species inhabiting phreatic waters and was found at only one well. Ecrobia ventrosa and Myosotella myosotis are species inhabiting brackish waters. These species were found at only two and one site respectively. Galba truncatula is the most widespre- 


\begin{tabular}{|c|c|c|c|c|c|c|c|c|c|c|c|c|c|c|}
\hline \multirow{27}{*}{$\begin{array}{l}\dot{0} \\
\stackrel{\Delta}{0} \\
\dot{\omega}\end{array}$} & ஜֶ & & & $\begin{array}{l}x \\
x \\
x\end{array}$ & & 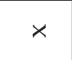 & & $\ddot{x}$ & & & & & & $m$ \\
\hline & ஸ็ & & & $\begin{array}{l}x \\
x \\
x\end{array}$ & & $\times$ & & & & & & & $\underset{x}{x}$ & $m$ \\
\hline & ন & & & $\stackrel{x}{x}$ & & $\stackrel{x}{x}$ & & $\stackrel{x}{x}$ & $\times$ & & & & $\stackrel{x}{x}$ & in \\
\hline & ले & & & & & $\times$ & & $\stackrel{x}{x}$ & $\underset{x}{x}$ & & & & $\times$ & $H$ \\
\hline & ల్ & & & & & & & & $\stackrel{x}{x}$ & & & & & - \\
\hline & $\approx$ & & & $\underset{x}{x}$ & & $\stackrel{x}{x}$ & $\stackrel{x}{x}$ & & & & & & & $n$ \\
\hline & $\vec{\lambda}$ & & & & & $\underset{x}{x}$ & & & & & & & & - \\
\hline & ㅇ & & & & & $x$ & & & & & & & & - \\
\hline & $\stackrel{\Rightarrow}{ }$ & $x$ & & & & & & & & & & & & - \\
\hline & $\stackrel{\infty}{\sim}$ & & & & & $\check{x}$ & & $\begin{array}{l}x \\
x \\
x\end{array}$ & & & & & & $N$ \\
\hline & $气$ & & & & & $x$ & & & $\vec{x}$ & & & & & $N$ \\
\hline & $\stackrel{0}{\longrightarrow}$ & & & & & $\times$ & & & $\underset{\substack{x \\
x}}{x}$ & & & & & N \\
\hline & $\stackrel{10}{\longrightarrow}$ & & $\underset{x}{x}$ & & & & & & & & & & & $r$ \\
\hline & 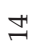 & & & $x$ & & $\ddot{x}$ & & & & & & & & N \\
\hline & $\stackrel{M}{2}$ & & & & & $\underset{x}{x}$ & & & & & & & & - \\
\hline & $\approx$ & & & & & & & & $x$ & & & & & - \\
\hline & $\exists$ & & & & & $\stackrel{x}{x}$ & & & & & & & & - \\
\hline & 윽 & & & & & $x$ & & & $\vec{x}$ & & & & & N \\
\hline & $a$ & & & $\times$ & & 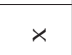 & 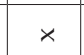 & $\underset{x}{x}$ & & & & & & $H$ \\
\hline & $\infty$ & & & $\stackrel{x}{x}$ & & & $\underset{x}{x}$ & 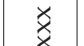 & & & & & & $m$ \\
\hline & $\wedge$ & & & $\underset{\substack{x \\
x}}{ }$ & & $x$ & $x$ & $\underset{x}{x}$ & $x$ & & & & & in \\
\hline & 6 & & & $\underset{\substack{x \\
x}}{x}$ & & & $\stackrel{x}{x}$ & & & & & $x$ & $\underset{x}{x}$ & $r$ \\
\hline & in & & & $\underset{x}{x}$ & & & $x$ & $x$ & & & & $x$ & $x$ & in \\
\hline & + & & & & & & $\underset{x}{x}$ & $\underset{x}{x}$ & rx & & & & & $\infty$ \\
\hline & $\infty$ & & $\underset{x}{x}$ & & & & & & & & & & & - \\
\hline & $\sim$ & & & & & $x$ & & & $\frac{x}{x}$ & & & & $\underset{x}{x}$ & $m$ \\
\hline & - & & & $\underset{x}{x}$ & & $x$ & & & & & & & $x$ & $m$ \\
\hline 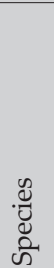 & 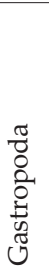 & 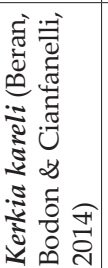 & 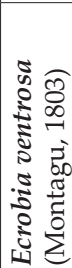 & 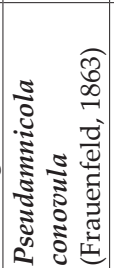 & 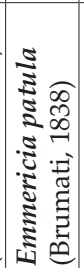 & 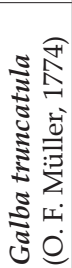 & 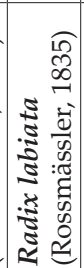 & 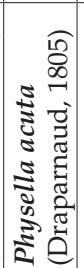 & 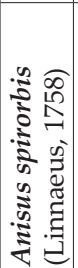 & 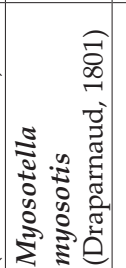 & $\frac{\sqrt[\pi]{5}}{\stackrel{.0}{\pi}}$ & 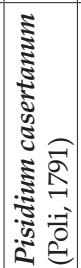 & 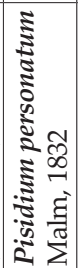 & 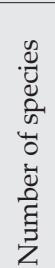 \\
\hline
\end{tabular}




\begin{tabular}{|c|c|c|c|c|c|c|c|c|c|c|c|c|c|c|}
\hline \multirow{27}{*}{$\begin{array}{l}\dot{0} \\
\stackrel{y}{0} \\
\dot{n}\end{array}$} & $\omega$ & - & $N$ & $\underset{\overparen{\Xi}}{\overparen{\Xi}}$ & $\widehat{\overparen{N}}$ & ๙ & 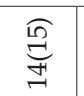 & $\stackrel{\infty}{\sim}$ & 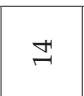 & - & & $H$ & $\triangleq$ & \\
\hline & Fे & & & & & $\overleftrightarrow{x}$ & & & & & & & & - \\
\hline & $\stackrel{\infty}{a}$ & & & & & $x$ & & & & & & & & - \\
\hline & भ & & & $\ddot{x}$ & & & $\ddot{x}$ & $\ddot{x}$ & & & & $\underset{x}{x}$ & & + \\
\hline & \& & & & 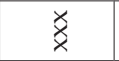 & 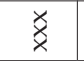 & & $\begin{array}{l}\underset{x}{x} \\
x\end{array}$ & & & & & & 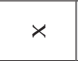 & $m$ \\
\hline & 通 & & & $x$ & & & & $\times$ & & & & & $x$ & $\underset{\widetilde{d}}{\overparen{d}}$ \\
\hline & $\mathscr{F}$ & & & $\widehat{x}$ & & $\underset{x}{x}$ & & $\underset{x}{x}$ & & & & & & त्ञ \\
\hline & ले & & & $\times$ & $\widetilde{x}$ & $\times$ & $\underset{x}{x}$ & $\underset{x}{x}$ & & & & & $\ddot{x}$ & $\stackrel{\overparen{f}}{\mathscr{f}}$ \\
\hline & ঙ্भ & & & $\times$ & & $\check{x}$ & & & & & & & & $N$ \\
\hline & 구 & & & $\times$ & $\stackrel{x}{x}$ & & $\begin{array}{r}\grave{x} \\
x \\
\end{array}$ & $\begin{array}{l}\underset{x}{x} \\
x\end{array}$ & & & & $\times$ & $\stackrel{x}{x}$ & 0 \\
\hline & $F$ & & & $\stackrel{x}{x}$ & & $\breve{x}$ & $\stackrel{x}{x}$ & $\times$ & & & & & $\times$ & in \\
\hline & of & & & $\begin{array}{r}x \\
x \\
\end{array}$ & & & $\begin{array}{r}\underset{x}{x} \\
\end{array}$ & & & & & & & $N$ \\
\hline & ले & & & & & 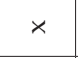 & & & & & & & & - \\
\hline & $\underset{\infty}{\infty}$ & & & & & $\check{x}$ & & 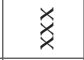 & & & & & & N \\
\hline & ह & & & & & 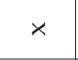 & & $\stackrel{x}{x}$ & $\underset{x}{x}$ & & & & & $\infty$ \\
\hline & $\stackrel{\pi}{m}$ & & & & & $\check{x}$ & & $\ddot{x}$ & $\underset{x}{x}$ & & & & $\underset{x}{x}$ & 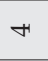 \\
\hline & $\infty$ & & & $\underset{x}{x}$ & & & $\times$ & $\underset{x}{x}$ & & & & & & $m$ \\
\hline & $\stackrel{12}{m}$ & & & & & & & & & $\ddot{x}$ & & & & $r$ \\
\hline & ले & & & & & $\times$ & & & $\ddot{x}$ & & & & & $N$ \\
\hline & 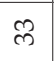 & & & & & & & & $\underset{x}{x}$ & & & & & - \\
\hline & $\tilde{\approx}$ & & & & & & & & $\times$ & & & & $\times$ & $N$ \\
\hline & $\vec{m}$ & & & & & $\times$ & & & & & & & & - \\
\hline & ৪ & & & $\underset{x}{x}$ & & 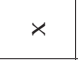 & & & $\times$ & & & & $\stackrel{x}{x}$ & $r$ \\
\hline & নे & & & $\grave{x}$ & & $\times$ & & & & & & & $\stackrel{x}{x}$ & $\infty$ \\
\hline & $\stackrel{\sim}{\sim}$ & & & & & $\overleftrightarrow{x}$ & & & & & & & $\times$ & $N$ \\
\hline & $\widehat{\curvearrowright}$ & & & & & $\times$ & & & & & & & & - \\
\hline & $\stackrel{\sim}{\sim}$ & & & $\widehat{x}$ & & $\check{x}$ & $\widehat{x}$ & & & & & & & స్త్ర \\
\hline $\begin{array}{l}\mathscr{8} \\
\tilde{0} \\
\tilde{0} \\
\text { के }\end{array}$ & 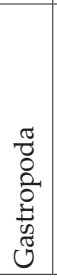 & 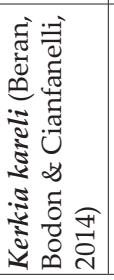 & 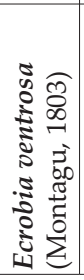 & 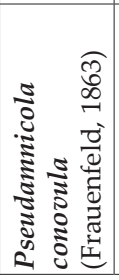 & 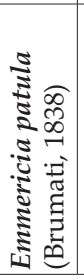 & 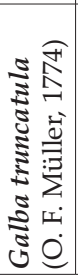 & 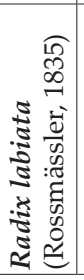 & 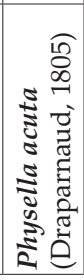 & 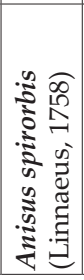 & 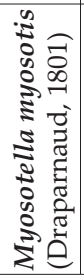 & 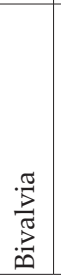 & 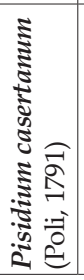 & 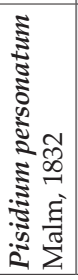 & 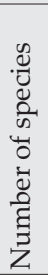 \\
\hline
\end{tabular}


ad species at this island and its occurrence was confirmed at 32 sites. Other 5 species (Pseudamnicola conovula, Radix labiata, Physella acuta, Anisus spirorbis, Pisidium personatum) were recorded from 14 to 19 sites (Tab. 1-2).

Most of the species (Myosotella myosotis, Galba truncatula, Radix labiata, Anisus spirorbis, Pisidium casertanum, P. personatum) belong to the category of common molluscs with extensive distribution ranges (Holarctic, Palaearctic, Euro-Siberian). Ecrobia ventrosa is restricted to Europe (sea coast) and Emmericia patula to Southern Europe. Only two species Kerkia kareli and Pseudamnicola conovula are molluscs known from restricted areas. Kerkia kareli has been known only from phreatic waters on Pag Island (BERAN et al., 2014) and during this research was found also on Rab Island. Pseudamnicola conovula is a freshwater species known from the Adriatic coast in Italy and Croatia (Croatian islands) (Giusti \& PezzoLi, 1980; Radoman, 1983; Beran, 2014; M. Bodon pers. comm., D. Delicado pers. comm.). Only one recorded species, Physella acuta, belongs to non-native and invasive species.

\section{DISCUSSION}

What is probably the first fairly detailed inventory of aquatic non-marine molluscs of Rab Island documented the occurrence of 11 species and this island belongs among the richest or at least the most investigated. Before this research the occurrence on Rab of only one species of non-marine aquatic mollusc was known, which is why it is not possible to compare this study with the results of previous surveys in the same area. Comparison is, however, possible with molluscan assemblages of several neighboring islands. Aquatic molluscs of the biggest Croatian island Krk were studied more than 50 years ago (BoLE, 1958) while the results of an inventory of the non-marine molluscs of Pag Island were published recently (BERAN, 2014). Seven species were recorded on Krk Island while Pag Island is inhabited by 14 species. However, at least in the case of the recently studied Pag Island it is possible to say that the freshwater mollusc communities are slightly different. On Rab Island species live much more frequently in small watercourses (Pseudamnicola conovula, Emmericia patula, Galba truncatula, Radix labiata, Pisidium casertanum, P. personatum). The reason for this is that there are a significantly more small but permanent watercourses. Conversely, at Pag Island, there are several molluscs common in stagnant waters (Bithynia tentaculata, Acroloxus lacustris, Stagnicola fuscus, Gyraulus crista), related to the relatively significant presence of such waters (the shallow lake Veliko Blato, the marshes Malo Blato). More significant differences can be found in the case of a comparison with the molluscan assemblages of the south-lying islands. One of these islands (Brač) was also examined recently (L. BERAN, unpublished records). There are almost no permanent streams and the occurrence of only two freshwater species (Ancylus fluviatilis, Musculium lacustre) was considered there. Neither of the recorded species was found on Pag Island or on Rab. Physella acuta is only non-native aquatic mollusc recorded on Rab Island and is also the first known record of the occurrence of a non-native aquatic mollusc from the northern Croatian islands.

In contrast, on some of the other islands there are much more suitable conditions for molluscs inhabiting brackish waters. On Rab Island only two common species were detected (Ecrobia ventrosa, Myosotella myosotis) at two and at one site, respectively, while on Pag four species have been detected (Hydrobia acuta, Ecrobia ventrosa, Truncatella subcylindrica, Myosotella myosotis) at several sites. Even on Brač Island which is very poor for freshwater molluscs there four brackish species occur (Heleobia stagnorum, Truncatella subcylindrica, Myosotela myosotis, Ovatella firminii) (L. BERAN, unpublished records). It is possible that more detailed research into the brackish habitats of Rab Island would 
have discovered more sites in which brackish molluscs occur and would also have increased the species richness.

Kerkia kareli, a species described in 2014 from Pag Island (BERAN et al., 2014), was found at one site. It probably also occurs at other sites of this island but during the research suitable sites for the collecting of samples were not found (wells). These results showed that this species is not endemic only to Pag Island and it is possible that it inhabits other Croatian islands. Boeters et al. (2014) described the similar species Arganiella tabanensis from Montenegro only several days after the description of Kerkia kareli was released. The description of $A$. tabanensis was published online 13.10.2014, while the date of publication of the description of $K$. kareli according to the editor of the Journal of Conchology is 8.10.2014 (G. Oliver, pers. comm.) This species is very similar to K. kareli and it is not to be excluded, that the species could be identical. In this case the name Arganiella tabanensis should be considered a junior synonym and the geographical range of $K$. kareli would be significantly greater than was expected. Nevertheless, the taxonomical position of $A$. tabanensis needs direct examination and a comparison of molecular data of both species is necessary. Meanwhile this species should be regarded as rare, occurring only on two Croatian islands. The more widespread (on the base of recent data) species K. jadertina is considered endangered in the IUCN Red List (BILANDZIJA \& JALZIC, 2010) while K. kareli has not been listed in the IUCN Red List yet and should be classified as vulnerable, which was also proposed by BERAN et al. (2014), or data deficient, due to the unclear taxonomical position of similar species mentioned above.

This research confirmed the fact that molluscan assemblages of Croatian islands are poorly known. Research could provide interesting and valuable information so further study of the non-marine aquatic molluscs of other Croatian islands is required.

\section{ACKNOWLEDGEMENTS}

I would like to thank my wife and our children for their help with field research and also for their patience. I am obliged to Helena Kaňková for preparing the map of Rab Island.

Received March 11, 2015

\section{REFERENCES}

Beran, L., 2014: Contribution to the knowledge of non-marine aquatic molluscan fauna (Gastropoda, Bivalvia) of Pag Island (Croatia). - Natura Croatica, 23(2), 303-313.

Beran, L., Bodon M. \& Cianfanelli S., 2014: Revision of "Hauffenia jadertina" Kuščer, 1933, and description of a new species from Pag Island, Croatia (Gastropoda: Hydrobiidae). - Journal of Conchology, 41(5), 585-601.

Bilandzija, H. \& JalzIC, B., 2010: Hauffenia jadertina. The IUCN Red List of Threatened Species. Version 2014.3. <www.iucnredlist.org>. Downloaded on 11 January 2015.

Boeters, H., Glöer P. \& Pešıć V., 2014: Arganiella tabanensis n. sp. from Montenegro (Mollusca: Gastropoda: Hydrobiidae). - Ecologica Montenegrina, 1(3), 131-139.

BoLe, J., 1958: Zoogeografski pregled malakofavne otoka Krka. [Zoogeographische übersicht der molluskenfauna der insel Krk]. - Biološki Vestnik, 6, 118-123.

Giusti, F. \& Pezzolı, E., 1980: Guide per il riconoscimento delle specie animali delle acque interne italiane. 8. Gasteropodi, 2. (Gastropoda: Prosobranchia: Hydrobioidea, Pyrguloidea). Collana del Progetto finalizzato "Promozione della qualità dell'ambiente", C.N.R., AQ/1/47, 67 pp.

Radoman, P., 1983: Hydrobioidea a superfamily of Prosobranchia (Gastropoda). I. Systematics. - Monographs Serbian Academy of Sciences and Arts, 57, 1-256. 


\title{
SUMMARY
}

\section{Non-marine aquatic molluscs (Gastropoda, Bivalvia) of Rab Island (Croatia)}

\author{
L. Beran
}

This paper presents the results of probably the first rather detailed malacological survey of Rab Island, which belongs among the Croatian islands situated in the northern Adriatic Sea. Aquatic molluscs of this island were previously poorly known and no inventory of nonmarine aquatic molluscs had been made. Altogether 11 species (Kerkia kareli, Ecrobia ventrosa, Pseudamnicola conovula, Emmericia patula, Galba truncatula, Radix labiata, Physella acuta, Anisus spirorbis, Myosotella myosotis, Pisidium casertanum, P. personatum) were found at 49 sites in 2012-2015. Brackish habitats, several upper sections of small rivulets and ditches with the positive occurrence of aquatic molluscs, were often inhabited by only one or two species while some brooks and rivulets were inhabited by more diversified molluscan assemblages with 3-6 species. Two recorded species, Ecrobia ventrosa and Myosotella myosotis, inhabit brackish waters while other species are freshwater molluscs that were found in springs, wells, ditches, rivulets and small brooks. Kerkia kareli, a species that inhabits phreatic waters and was described in 2014 from Pag Island, was also found. The non-native Physella acuta was recorded from many sites and this find is probable the first known occurrence of a non-native aquatic mollusc from the northern Croatian islands. Before this research the occurrence of only one species of non-marine aquatic molluscs was known from this island. The research showed that this island belongs among the richest or at least the best researched of the Croatian islands. 